\title{
Preparation of Nanometer Cobalt Particles by Polyol Reduction Process and Mechanism Research
}

\author{
Liu Biao ${ }^{1}$, Guan Jian-guo ${ }^{1, *}$, Wang Qi ${ }^{1,2}$ and Zhang Qing-jie ${ }^{1}$ \\ ${ }^{1}$ State Key Laboratory of Advanced Technology for Materials Synthesis and Processing, Wuhan University of Technology, \\ Wuhan 430070, P. R. China \\ ${ }^{2}$ School of Materials Science and Engineering, Jinan University, Jinan 250022, P. R. China
}

\begin{abstract}
By the liquid-reduction process, the nanometer spherical cobalt powders with $f c c$ structure and a crystal size of $10 \mathrm{~nm}$, are prepared using the regents of cobaltous salt as precursor and 1,2-propanediol as reducing agent. XRD, TEM are applied to characterize the phase and morphology of the as-prepared products. Both the reaction process and mechanism of the polyol reduction process are discussed preliminary through FT-IR spectra of the systems before and after reduction.
\end{abstract}

(Received December 22, 2004; Accepted June 3, 2005; Published August 15, 2005)

Keywords: nanometer particles, cobalt, polyol reduction process, mechanism

\section{Introduction}

With the prosperity in nano-technology, the nanometer magnetic materials have displayed an entirely new applications of prospects for the field of functional materials because of their novel and unique properties. Cobalt nanocrystals have displayed their wonderful properties in magnetic, electronic, catalytic fields, their applications to magnetic recording materials, permanent magnetic materials and radar absorbing materials are playing an increasingly important role. ${ }^{1)}$ So far, the main methods of preparing nanometer Co powders are microemulsion synthesis, 2,3$)$ vapour deposition, ${ }^{4)}$ reduction by hydrazine hydrate ${ }^{5)}$ or by sodium (or potassium) borohydride, ${ }^{6}$ decomposition of cobalt carbon$\mathrm{yl}^{7)}$ By these processes, the single phase of nanometer Co powders is obtained, or the as-prepared samples are amorphous. Luna et al. ${ }^{8)}$ reported that Co nanoparticles with $h c p$ structure were synthesized by polyol reduction of $\mathrm{Co}^{2+}$, using 1,2-hexadecandiol rather than ethylene glycol. Hinotsu et $a{ }^{9}{ }^{9}$ ) also reported that the micron size Co particles synthesized in ethylene glycol had a mixture of $f c c$ and $h c p$ phase by polyol process. In this paper, we have found on the basis of the above-metioned researches and other researches in preparing $\mathrm{Ni}$ nanoparticles by polyol reduction process $^{10,11)}$ that the $f c c$ Co nanoparticles is prepared with the cobaltous salt as precursor and the 1,2-propanediol as reducer. This process is proved to be very simple, and pure non-oxidized products are obtained. XRD, TEM are applied to characterize the phase and morphology of the as-prepared products. A preliminary discussion about the reaction process and mechanism of the polyols reduction process is done through FT-IR spectra of the systems before and after the reduction.

\section{Experimental Section}

\subsection{Materials}

Cobaltous chloride hexahydrate (A.P., Shanghai Hengxin

*Corresponding author, E-mail: guanjg@mail.whut.edu.cn,

Tel: 86-27-87218832, Fax: 86-27-87879468
Chemical Reagent Co. Ltd.), 1,2-propanediol (A.P., Zhengzhou No. 3 Chemical Reagent Ind.), sodium hydrate (A.P., Tianjin No. 3 Chemical Reagent Ind.) were used for the reduction process. Ethanol (A.P.) or acetone (A.P.) and deionized water were used to wash the products.

\subsection{Preparation}

The preparation of Co nanoparticles was performed in a three-necked flask. $1.19 \mathrm{~g}(0.005 \mathrm{~mol})$ of cobaltous chloride hexahydrate and $0.5 \mathrm{~g}(0.0125 \mathrm{~mol})$ sodium hydrate were dissolved in $14.5 \mathrm{~mL}$ of 1,2-propanediol respectively. Both propanediol solutions above were mixed together, while stirring and heating to boiling. Nitrogen bubbling was maintained during the reactions. The particles formation was manifested by a dark gray coloration of the dispersion. The Co particles were separated from dispersion by centrifugation and washed by deionized water to $\mathrm{pH}=7$, then ethanol or acetone was used to purge the surface of particles. The resulting particles are dried in a vacuum dryer at $50^{\circ} \mathrm{C}$ for $24 \mathrm{~h}$.

\subsection{Characterization}

The structure and morphology of the as-prepared samples were characterized by an X-ray powder diffraction (XRD) (Rigaku, D/Max-2400, $\mathrm{Cu} \mathrm{K} \alpha$ radiation, $\lambda=0.15405 \mathrm{~nm}$ ) technique and a transmission electron microscopy (TEM) (Hitachi, H-800). The crystalline size was calculated by Scherrer equation $(D=K \lambda / \beta \cos \theta$, in which $K=1)$, the step length for step scan was $0.02^{\circ}$. The FT-IR spectra were measured by a infrared spectrometer (Nicolet, 60SXB).

\section{Results and Discussion}

\subsection{Crystal phase and size characterization}

The XRD results for Co particles as-prepared by polyol reduction process is shown in Fig. 1. It can be observed that the characteristic peaks for metallic Co are evident, and no peaks for $\mathrm{CoO}$ or $\mathrm{Co}_{2} \mathrm{O}_{3}$ are found. The crystal structure of the Co particles is $f c c$ ( $\beta$-Co, JCPDS 15-806). The very weak peaks near $2 \theta=42^{\circ}$ and $47^{\circ}$ belong to $h c p$ Co particles $(\alpha$ Co, JCPDS 5-0727) according to the results by Hinotsu et 


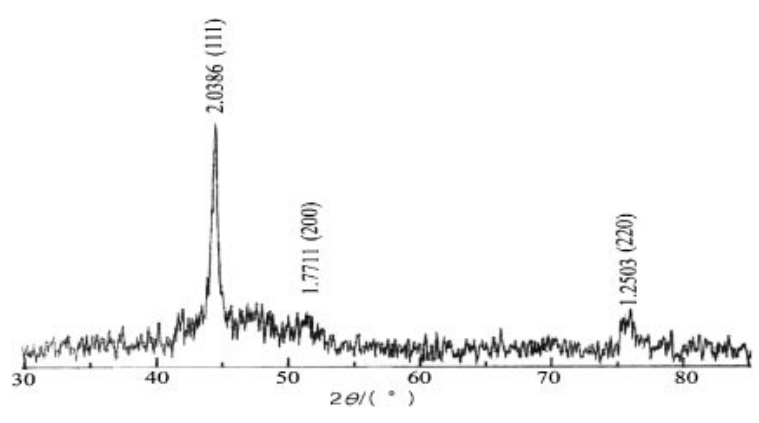

Fig. 1 XRD pattern of Co particles as-prepared by polyol reduction process.

al. ${ }^{9)}$ Generally, the bulk metallic Co is ordinarily $\alpha$ phase, and the reversible transformation of $\alpha$ to $\beta$ for metallic Co happens at $417^{\circ} \mathrm{C}$. However, the as-prepared Co nanoparticles by physical method (such as vapor deposition, gas evaporation) are different from the bulk matellic Co with hcp structure, which are unique $f c c$ structure with the particle size of $5-35 \mathrm{~nm}$.

It can be seen from the XRD patterns that the peak of (200) crystal face for $\beta$-Co $(d=0.17711 \mathrm{~nm})$ is very weak, in contrast to the broad peak of (111) face. These results indicate that the crystal grains are very fine. The size of the $\beta$ Co crystal are estimated from XRD line broadening of the peaks at $44.2^{\circ}$ according to Scherrer equation, and values of $19 \mathrm{~nm}$ is obtained.

\subsection{Morphology}

Typical TEM picture of $\beta$-Co particles is shown in Fig. 2(a). It can be seen from TEM picture that the particles are nearly spherical and well dispersed. The particle size is even, the maximum size is $13 \mathrm{~nm}$, and the size of the vast majority of particles is $10 \mathrm{~nm}$. Figure 2(b) is the polycrystalline diffraction rings of $\beta$-Co particles. Because of the $f_{c c}$ structure of $\beta$-Co particles, corresponding indices of crystal plane for the diffraction rings are (111), (200), (220) from the inner to outer ones.

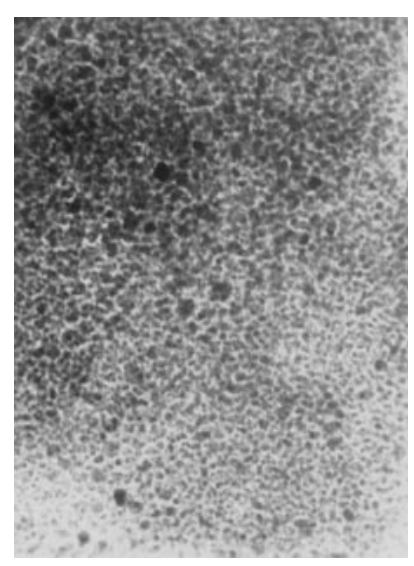

(a) $\times 150000$

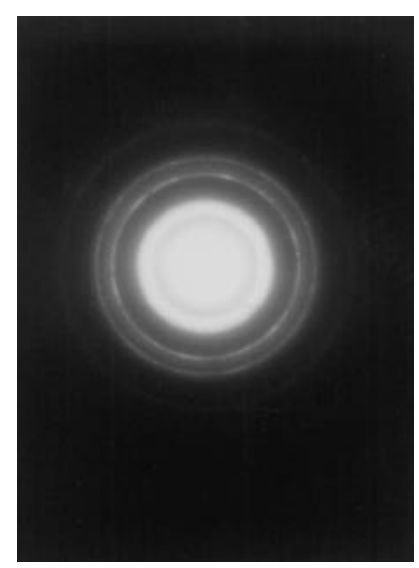

(b)
Fig. 2 TEM pictures of nanometer cobalt particles (a) and poly-crystal diffraction rings (b)

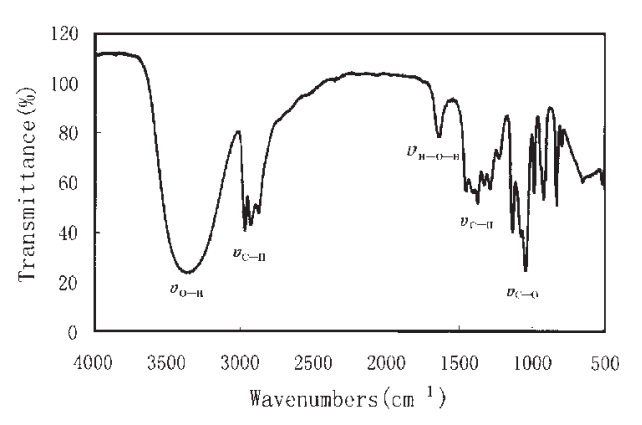

Fig. 3 FT-IR spectra of system before reduction.

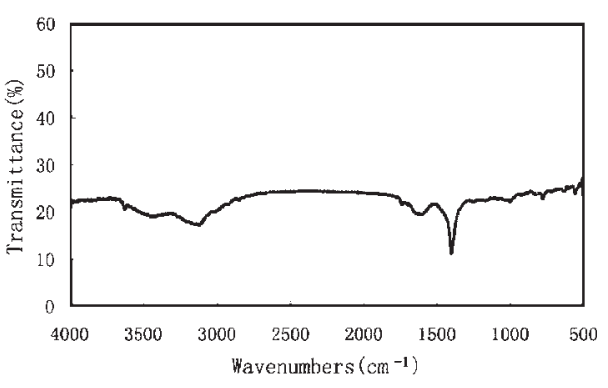

Fig. 4 FT-IR spectra of intermediate.

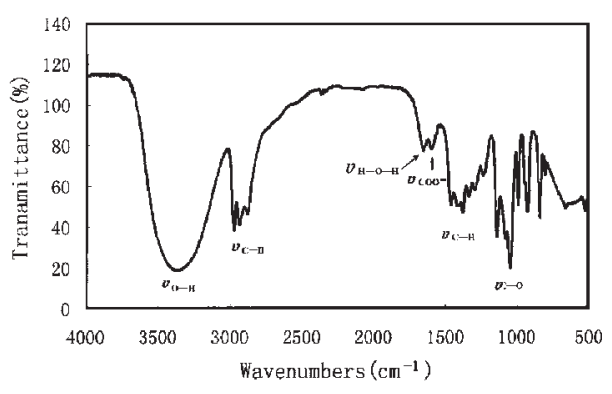

Fig. 5 FT-IR spectra of system after reduction (liquid).

\subsection{Reduction process and mechanism}

Figures 3, 4 and 5 are FT-IR spectra of the systems before and after reduction respectively. The absorption spectra in 3346.2, 2970.9, 1459.2, 1377.2, 1137.8, 1046.0, 990.9, 923.7, $838.2 \mathrm{~cm}^{-1}$ are the typical spectra for 1,2-propanediol. The reducing agent, 1,2-propanediol, is greatly excessive. When $\mathrm{CoCl}_{2}$ dissolves in 1,2-propanediol, a transparent solution with pinkish-red color is obtained. Because $\mathrm{Co}^{2+}$ ions are main parts of coordination compound, the coordination compound with octahedron structure whose coordination number is 6 can easily form. The solution appeared pinkishred because of $d-d$ electronic transition. After mixing the $\mathrm{Co}^{2+}$ and $\mathrm{NaOH}$ solution, a transition complex flocculent deposition $\mathrm{Co}(\mathrm{OH}) \mathrm{Cl}$ with blue color firstly generated $\left(\mathrm{Co}^{2+}+\mathrm{Cl}^{-}+\mathrm{OH}^{-} \rightarrow \mathrm{Co}(\mathrm{OH}) \mathrm{Cl} \downarrow\right)$, then changed into intermediate precipitates with pinkish-red coloration with the increase of temperature $\left(\mathrm{Co}(\mathrm{OH}) \mathrm{Cl}+\mathrm{OH}^{-} \rightarrow\right.$ $\left.\mathrm{Co}(\mathrm{OH})_{2} \downarrow+\mathrm{Cl}^{-}\right)$. The precipitates had the same crystal with $\beta-\mathrm{Co}(\mathrm{OH})_{2}$ (see Fig. 4 and Fig. 6, JCPDS 30-443). In Fig. 4 , the absorption spectra in $3123,1606 \mathrm{~cm}^{-1}$ probably belong to the absorption of $\mathrm{OH}^{-}$in inorganic solid, and the absorption spectra in $1401 \mathrm{~cm}^{-1}$ likely relates to the bonds of 


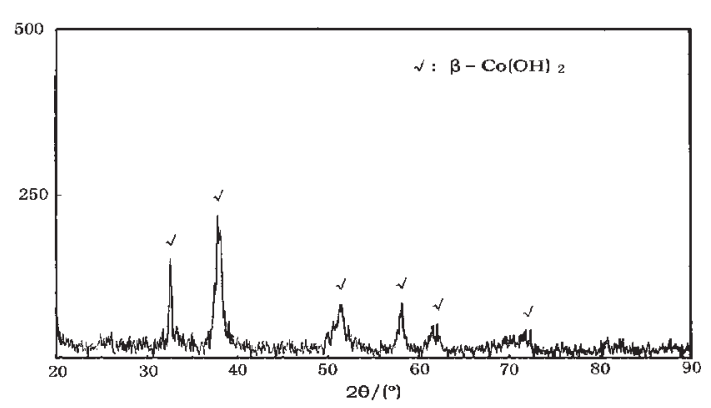

Fig. 6 XRD spectra of intermediate.

metal ions and $\mathrm{OH}^{-}$. The intermediate may be just like a storehouse of $\mathrm{Co}^{2+}$ which continuously releases $\mathrm{Co}^{2+}$ ions into the liquid phase so that they can be reduced. The solution of $\mathrm{Co}(\mathrm{OH})_{2}$ in water is $K_{\mathrm{sp}}=1.6 \times 10^{-18}$, and that of 1,2 propanediol is also very small, the reduction reaction is controlled by the solubility product of the intermediate. Lastly the Co nanoparticles are formed through nucleation, crystallization and growth processes. The strong absorption spectrum in $1610-1569 \mathrm{~cm}^{-1}$ for $\mathrm{COO}^{-}$is seen from the IR spectrum of the systems after reduction, which means that 1,2-propanediol is partly oxidized.

As a summary of the present results, at least three steps are taken for the preparation of Co nanoparticles by liquidreduction, solution of precursors in 1,2-propanediol, reduction of $\mathrm{Co}^{2+}$ ions released by intermedium and nucleation/ growth of crystal.

It is noteworthy that $\mathrm{NaOH}$ is of importance during the liquid-reduction process. Firstly, $\mathrm{NaOH}$ reacts with $\mathrm{CoCl}_{2}$ to form the active intermediate so that the $\mathrm{Co}^{2+}$ ions can be activated effectly. The active intermediate $\mathrm{Co}(\mathrm{OH})_{2}$ is the key factor which controls the oxidation-reduction reaction by continuously releasing $\mathrm{Co}^{2+}$ ions into the liquid phase so that they can be reduced. Secondly, according to Lewis Acidalkali Theory, acid and alkali are catalysts for many reactions. The central point for them is the shift of protons, which can decrease the activation energy and accelerate the reactions. As a strong alkali, ${ }^{12)} \mathrm{NaOH}$ can provide conductive electrons, which may be related to cut down the activation energy of reduction by electron migration, then eliminate the kinetic barrier by surface adsorption so that the reduction can perform on the surface.

\section{Conclusions}

We have devised a simple liquid reduction method for the preparation of Co nanoparticles. The $\beta$-Co nano-particles can be prepared by polyols reduction process in 1,2-propanediol. The particles are nearly spherical and well dispersed, the size of the vast majority of particles is about $10 \mathrm{~nm}$.

The preparation of Co nanoparticles by polyols reduction process is an oxidation-reduction reaction. The result of oxidation-reduction process is that $\mathrm{Co}^{2+}$ is reduced and 1,2propanediol is oxidized, XRD and FT-IR spectra can give a direct evidence. The preparation process is made by three steps, that is, solution of precursors in 1,2-propanediol, reduction of $\mathrm{Co}^{2+}$ ions released by intermedium and growth of crystal. $\mathrm{NaOH}$ takes an important role during the polyols reduction process.

\section{Acknowledgement}

This work was financed by the National Natural Science Foundation of China for the Key Projects (No. 29904005) and the 863 High Technology Research Project (granted Nos. 2001AA339020 and 2002AA305302) from the Ministry of Science and Technology of China.

\section{REFERENCES}

1) P. Vargas, D. Altbir and J. d'Albuquerquee Castro: J. Magn. Magn. Mater. 226-230 (2001) 603-605.

2) J. P. Chen, K. M. Lee, C. M. Sorensen, K. J. Klabunde and G. C. Hadjipanayis: J. Appl. Phys. 75 (1994) 5876-5878.

3) J. P. Chen, C. M. Sorensen, K. J. Klabunde and G. C. Hadjipanayis: Phys. Rev. B 51 (1995) 11527-11532.

4) S. Gangopadhyay, G. C. Hadjipanayis, C. M. Sorensen and K. J. Klabunde: IEEE Trans. Magn. 28 (1992) 3174-3176.

5) H. Li and W. Gong: Acta Phys. Sinica 40 (1991) 1356-1363.

6) C. P. Gibson and K. J. Putzer: Science 267 (1995) 1338-1340.

7) H. Zheng, J. Zeng and J. Liang: Acta Metall. Sinica 35 (1999) 837-840.

8) C. Luna, M. P. Morales, C. J. Sema and M. Vázquez: Mater. Sci. Eng. C 23 (2003) 1129-1132.

9) T. Hinotsu, B. Jeyadevan, C. N. Chinnasamy, K. Shinoda and K. Tohji: J. Appl. Phys. 95 (2004) 7477-7479.

10) M. S. Hegde and D. Laicher: Solid State Ionics 93 (1997) 33-50.

11) P. Li, J. G. Guan, Q. J. Zhang, W. Y. Zhao and R. Z. Yuan: Mater. Sci. Technol. 9 (2001) 259-262.

12) G. Rong and K. Su: Theory of College Organic Chemistry. (China Chemical Industry Press, 2000). 